\title{
Extended RAS Mutational Status Analysis in Circulating Tumor DNA from Patients with Advanced Colorectal Cancer in Daily Clinical Practice. The Franco-British Institute Experience and Recommendations
}

Laurence Heuls ${ }^{1}$, Nathalie Perez Staub ${ }^{2}$, Ida Iurisci ${ }^{1,2}$, Perrine Goyer ${ }^{3}$, Arnaud Saget ${ }^{4}$, Hubert Richa ${ }^{5}$, Hélène Marijon $^{2}$, Linda Dainese ${ }^{6}$, Annette K Larsen ${ }^{7,8}$, Aimery De Gramont ${ }^{1,2,7,8}$ and Benoist Chibaudel ${ }^{* 1,2,7,8}$

${ }^{1}$ Department of Clinical and Translational Cancer Research Unit, Franco-British Institute, Levallois Perret, France

${ }^{2}$ Department of Medical Oncology, FrancoBritish Institute, Levallois Perret, France

${ }^{3}$ Department of Gastrointestinal Surgery, Clinique Turin, France

${ }^{4}$ Department of Gastrointestinal Surgery, Clinique Ambroise Paré, Neuilly SurSeine, France

${ }^{5}$ Department of Gastrointestinal Surgery, FrancoBritish Institute, Levallois Perret, France

${ }^{6}$ Department of Center of Anatomic Pathology, Institut De Pathologie De Paris, Malakoff, France

${ }^{7}$ Depatment of Cancer Biology and Therapeutics, Centre De Recherche Saint-Antoine (CRSA), France

${ }^{8}$ Institut National de la Santé et de la Recherche Médicale (INSERM) U938, Paris, France

Received: 制 June 25, 2018; Published: 制July 13, 2018

*Corresponding author: Benoist Chibaudel, Department of Medical Oncology, Franco British Institute, France

\section{Abstract}

Background : The development of liquid biopsies could help clinicians to shorten treatment decision and allow longitudinal molecular monitoring. We report here our experience of using the Biocartis Idylla ${ }^{\mathrm{TM}}$ test for patients with advanced colorectal cancer in daily clinical practice.

Patients and Methods: This program prospectively enrolled patients with advanced colorectalcancer at the Department of Medical Oncology of the Franco-British Institute, Levallois-Perret,France.Theprimary objective was to evaluate the feasibilty of the Idylla ${ }^{\mathrm{TM}}$ testing in daily clinical practice.

Results: From November 17, 2017 to June 12,2018, 50 patients were tested. The median time to circulating tumor (ct) extended mutational status was 4.8 hours (95\% CI:4.7-5.1). Among 37 (74.0\%) patients with paired plasma and tumor samples available, the overall agreement rate was $73.0 \%$. In patients with previously untreated metastatic liver disease $(\mathrm{n}=9 ; 18 \%)$, the overall agreement rate between paired plasma and tumor samples was $100.0 \%$, and the weighted K coefficient was 1.00 (95\% CI: 1.00-1.00).

Conclusion : Extended RAS mutational status analysis in circulating tumor DNA from patients with advanced colorectal cancer is feasible and greatly helpful for daily clinical practice. We recommend restricting ctDNA testing with Idylla ${ }^{\mathrm{TM}}$ to patients with previously untreated liver metastatic disease.

Keywords: Colorectal Cancer; Liquid Biopsy; KRAS; NRAS; BRAF

Abbrevations: EGF: Epidermal Growth Factor; MoAb: Monoclonal Antibodies; KRAS: Kirsten Rat Sarcoma Viral Oncogene Homolog; ECD: Extra Cellular Domain; ctDNA: Circulating Tumor DNA; CT: Circulating Tumor

\section{Introduction}

Systemic therapy is the standard care for patients with unresectable metastatic colorectal cancer (CRC). Cetuximab and panitumumab are IgG monoclonal antibodies (MoAb), which competitively inhibit the binding of epidermal growth factor (EGF) and other ligands to EGF receptor (EGFR) and downregulate EGFR, resulting in inhibition of cell survival, growth, proliferation, and transformation. Tumor molecular testing for Kirsten rat sarcoma viral oncogene homolog (KRAS) and Neuroblastoma RAS viral oncogene homolog (NRAS) tumor genes is mandatory in order to select MCRC patients who may benefit from anti-EGFR MoAb therapy [1]. In 2014, the mean time to obtain the RAS genotyping test report in France was 25 days, and for $25 \%$ of the patients it was more than one month [2]. The murine sarcoma viral (v-Raf) oncogene homolog B (BRAF) is a human gene that encodes BRAF, a protein involved in sending signals inside cells, which are involved in directing cell growth. The BRAF V600E mutation is found in $10 \%$ of patients with colorectal adenocarcinoma [3] and BRAF mutant tumors are more frequently right-sided, mucinouswith peritoneal spreadand microsatellite instability [4].

The extracellular domain (ECD) of EGFR p.S492 mutation has been described as an acquired resistance to cetuximab [5]. The 
development of liquid biopsies could help clinicians to shorten treatment decision and allow longitudinal molecular monitoring. The concordance between plasma and tumor RAS mutational status ranges from $84 \%$ to $100 \%$ depending on different techniques (duplex dPCR, allele specific quantitative PCR, BEAMing [OncoBEAM] or NGS BPER) [6-13]. We report here our experience of using the Biocartis Idylla ${ }^{\mathrm{TM}}$ test for daily clinical practice in order to improve care in patients with advanced CRC.

\section{Material and Methods}

\section{Study Design and Population}

This program prospectively enrolled patients with advanced CRC at the Department of Medical Oncology of the Franco-British Institute, Levallois-Perret, France. Clinical research study nurse was responsible for this program. A signed and dated informed consent form from each patient was required before enrollment.

\section{Methods}

The blood sampling was done in the out patient cancer unit, close to the translational research laboratory. EDTA tubes were centrifuged at $1500 \mathrm{~g}$ for 10 minutes within 4 hours after blood samples collection, the plasma was recovered, and a second centrifugation was performed at $16000 \mathrm{~g}$ for one minute. Circulating tumor DNA (ctDNA) was extracted and amplified from $1 \mathrm{ml}$ of blood plasma. The Idylla ${ }^{\mathrm{TM}}$ ctKRAS Mutation Assay is a molecular assay for the qualitative detection of 21 mutations in codons 12, 13, 59, 61,117and 146 of the KRAS gene. The Idylla ${ }^{\text {TM }}$ ctNRAS-BRAFEGFR S492R Mutation Assay is a molecular assay for the semiquantitative detection of 18 mutations in codons $12,13,59,61,117$, and 146 of the NRAS gene, five mutations in codon 600 of the BRAF gene and two mutations in codon 492 of the EGFR gene. Biocartis Idylla $^{\mathrm{TM}}$ platform is a CE-marked IVD in Europe.

\section{Data Collection}

Individual patient-level clinical data and tumor mutational RAS and BRAF assessment were collected in DxCare software (C2013 Medasys S.A., Clamart, France). Circulating tumor DNA information was collected using the Idylla ${ }^{\mathrm{TM}}$ Explore, a web-based application that allows analyzing data by visualization of PCR curves from the Idylla ${ }^{\mathrm{TM}}$ tests results, Cq values per target, and direct access to console results report.

\section{Statistical Analysis}

The primary objective was to evaluate the feasibility of the Idylla $^{\mathrm{TM}}$ testing in daily clinical practice. Secondary objectives were:

a) To evaluate the accuracy of RAS and BRAF status between paired plasma and tumor samples

b) To compare the time to molecular mutational diagnosis between tumor and plasma techniques in patients with initially unknown tumor status

c) To evaluate the incidence of neomutations in patients with wild-type CRC treated with anti-EGFR MoAb using a serial tracking of KRAS, NRAS, BRAF, and EFGR mutations

d) To evaluate the incidence of mutation loss in patients with mutated RAS or BRAF CRC.

The feasibility of the Idylla ${ }^{\mathrm{TM}}$ testing was calculated using the time from blood sample collection to the Idylla ${ }^{\mathrm{TM}}$ processing and results. The accuracy was calculated using the interrate agreement kappa(K) coefficient [14] with its 95\% circulating tumor). The strength of agreement was interpreted according to $\mathrm{K}$ value as follows: poor $(<0.20)$, fair $(0.21-0.40)$, moderate $(0.41-0.60)$, good (0.61-0.80), and very good (0.81-1.00). All analyses were performed using MedCalc Statistical Software version 18.2.1 (MedCalc Software bvba, Ostend, Belgium, http://www.medcalc. org; 2018).

\section{Results}

\section{Patient Characteristics}

From November 17, 2017 to June 12,2018, 50 patients with advanced CRC had their tumor genotyped by the Idylla ${ }^{\mathrm{TM}}$ test. This testing was proposed to $30(60.0 \%)$ patients with unknown or partially known RAS/BRAF tumor status and to 20 (40.0\%) patients with known status for the RAS and BRAF tumor genes (Table1) and (Figure 1). The mean age was 67.9 years (range: 29.7-93.4). The IdyllaTM test was done in 33(66.0\%) patients before starting a new treatment sequence and in 17 (34.0\%) patients with ongoing treatment sequence.

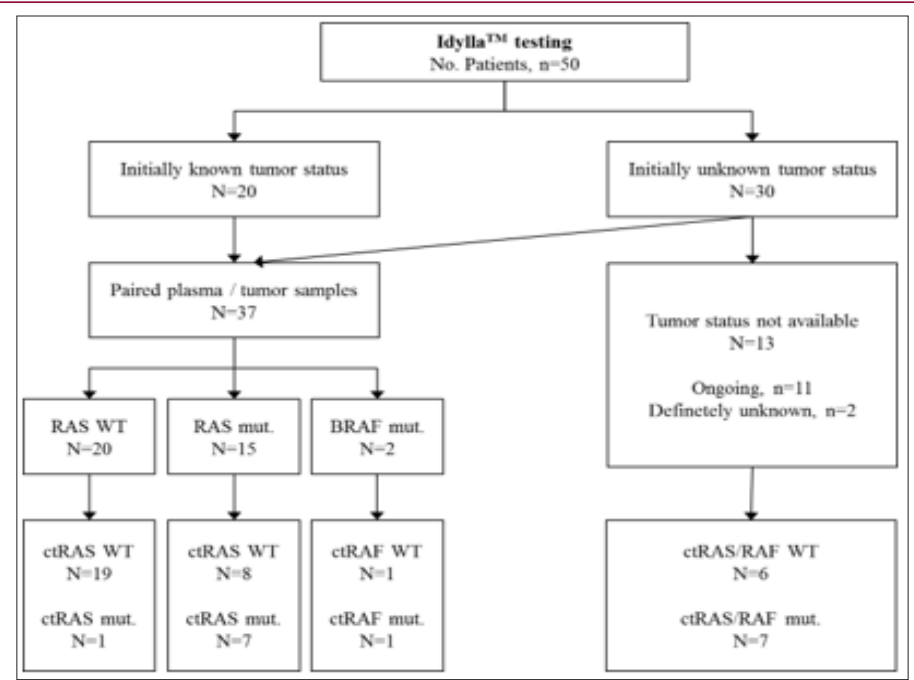

Figure 1: Flowchart. 
Table 1: Patient characteristics, n (\%).

\begin{tabular}{|c|c|c|c|c|c|c|}
\hline & \multicolumn{2}{|c|}{$\begin{array}{c}\text { All } \\
\mathbf{n = 5 0}\end{array}$} & \multicolumn{2}{|c|}{$\begin{array}{l}\text { Unknown or Partial Tumor Mutational } \\
\text { status } \\
\qquad=30\end{array}$} & \multicolumn{2}{|c|}{$\begin{array}{l}\text { Known Tumor Mutational Status } \\
\qquad \mathbf{n}=\mathbf{2 0}\end{array}$} \\
\hline & $\mathbf{n}$ & $\%$ & $\mathbf{n}$ & $\%$ & $\mathbf{n}$ & $\%$ \\
\hline \multicolumn{7}{|l|}{ Gender } \\
\hline Male & 28 & 56 & 13 & 43.3 & 15 & 75 \\
\hline Female & 22 & 44 & 17 & 56.7 & 5 & 25 \\
\hline \multicolumn{7}{|l|}{ Age } \\
\hline$<70$ & 28 & 56 & 17 & 56.7 & 11 & 55 \\
\hline$\geq 70$ & 22 & 44 & 13 & 43.3 & 9 & 45 \\
\hline \multicolumn{7}{|c|}{$\begin{array}{l}\text { RAS/BRAF tumor } \\
\text { mutational status }\end{array}$} \\
\hline Wild-type & 20 & 40 & 8 & 26.7 & 12 & 60 \\
\hline Mutant & 17 & 34 & 9 & 30 & 8 & 40 \\
\hline Unknown & 13 & 26 & 13 & 43.3 & 0 & 0 \\
\hline \multicolumn{7}{|l|}{$\begin{array}{l}\text { Primary tumor } \\
\text { location }\end{array}$} \\
\hline Right & 13 & 26 & 10 & 33.3 & 3 & 15 \\
\hline Left & 25 & 50 & 14 & 46.7 & 11 & 55 \\
\hline Rectum & 11 & 22 & 5 & 16.7 & 6 & 30 \\
\hline $\begin{array}{l}\text { Unknown or } \\
\text { missing }\end{array}$ & 1 & 2 & 1 & 3.3 & 0 & 0 \\
\hline \multicolumn{7}{|c|}{$\begin{array}{l}\text { Prior primary } \\
\text { tumor resection }\end{array}$} \\
\hline No & 25 & 50 & 19 & 63.3 & 6 & 30 \\
\hline & 25 & 50 & 11 & 36.7 & 14 & 70 \\
\hline \multicolumn{7}{|c|}{$\begin{array}{l}\text { No. of metastatic } \\
\text { sites }\end{array}$} \\
\hline 0 & 3 & 6 & 2 & 6.7 & 1 & 5 \\
\hline 1 & 23 & 46 & 17 & 56.7 & 6 & 30 \\
\hline$\geq 2$ & 24 & 48 & 11 & 36.6 & 13 & 65 \\
\hline \multicolumn{7}{|l|}{ Metastatic sites } \\
\hline Liver & 34 & 68 & 20 & 66.7 & 14 & 70 \\
\hline Lung & 19 & 38 & 9 & 30 & 10 & 50 \\
\hline Nodes & 8 & 16 & 3 & 10 & 5 & 25 \\
\hline Peritoneum & 14 & 28 & 8 & 26.7 & 6 & 30 \\
\hline
\end{tabular}

\section{Feasibility of the Idylla ${ }^{\mathrm{TM}}$ Testing}

Seventy three samples analyses (including longitudinal testing) were performed. ctKRAS mutation assay was done in 73 samples and ctNRAS/BRAF/EGFR mutation assay was performed for 60 samples. The median time from blood sample collection to the Idylla ${ }^{\mathrm{TM}}$ processing was 34 minutes (95\% CI: 29-39). The median time to ctKRAS results and to full ctKRAS/NRAS/BRAF/EGFR results were 2.8 hours (95\% CI: $2.7-2.9$ ) and 4.8 hours (95\% CI: 4.7-5.1), respectively.

\section{Time to Molecular Mutational Diagnosis: Plasma vs Tumor}

In $30(60 \%)$ patients with initially unknown tumor status, the median time to molecular profile using liquid biopsies was 4.8 hours (range:2.5-65.6). At the time of analysis, the tumor molecular profile was obtained in $17(56.7 \%)$ patients, in whom the median time to a full molecular diagnosis was 56.0 days (95\% CI: 35.183.9). 
Accuracy of RAS and BRAF Status Between Paired Plasma and Tumor Samples

Among $37(74.0 \%)$ patients with paired plasma and tumor samples available, $27(73.0 \%)$ had a concordant molecular profile between plasma and tumor. Positive, negative, and overall agreement rates were $47.0 \%, 95.0 \%$ and $73.0 \%$, respectively. The weighted $\mathrm{K}$ coefficient was 0.44 (95\% CI: 0.17-0.70). Among 10 (20\%) patients with discordant results between plasma and tumor, five patients had single extra-liver metastatic site (lung, $n=3$; peritoneum, $n=2$ ) and five patients received prior therapy for metastatic disease (Table 2). When selecting only patients with paired plasma and tumor samples with previously untreated metastatic liver disease $(\mathrm{n}=9 ; 18 \%)$, there were no discordant results between plasma and tumor. The positive, negative and overall agreement rates were $100.0 \%$, and the weighted K coefficient was 1.00 (95\% CI: 1.001.00).

Table 2: Discordant results, $\mathrm{n}=10$.

\begin{tabular}{|c|c|c|c|c|c|c|c|c|}
\hline Patient & Tumor & Plasma & Sidedness & $\begin{array}{c}\text { Primary } \\
\text { Tumor }\end{array}$ & Time to Met. & No. of Sites & Liver & No. of Lines \\
\hline BR260332-01 & Mut KRAS & ctWT & Unknown & Not resected & Synchronous & 1 & No & 1 \\
\hline CM171250-01 & Mut KRAS & ctWT & Right & Resected & Synchronous & 3 & Yes & 2 \\
\hline DF280249-01 & Mut KRAS & ctWT & Right & Not resected & Synchronous & 2 & Yes & 1 \\
\hline LJ080777-01 & Mut KRAS & ctWT & Left & Resected & Synchronous & 3 & Yes & 3 \\
\hline L0080230-01 & Mut KRAS & ctWT & Left & Resected & Synchronous & 1 & No & 1 \\
\hline DJ280440-01 & Mut KRAS & ctWT & Rectum & Resected & Metachronous & 1 & No & 0 \\
\hline GD060951-01 & Mut KRAS & ctWT & Rectum & Not resected & Synchronous & 1 & No & 1 \\
\hline TM311234-01 & Mut KRAS & ctWT & Rectum & Resected & Metachronous & 3 & Yes & 3 \\
\hline JP220454-01 & Mut BRAF & ctWT & Left & Resected & Metachronous & 1 & No & 3 \\
\hline YM221242-01 & WT & ctMut KRAS & Left & Resected & Metachronous & 2 & Yes & 1 \\
\hline
\end{tabular}

\section{Neomutations in Wild-Type Crc Patients}

Of the nine (18\%) patients with wild-type RAS/RAF CRC treated with anti-EGFR MoAb using a serial tracking of KRAS, NRAS, BRAF and EFGR mutations, two (22.2\%) had neomutations. The first patient was treated with FOLFIRI-cetuximab as firstline therapy for right-sided unresectable metastatic CRC. A ctNRAS G12A/V neomutation was not confirmed in further liquid biopsy and was contemporary of partial response (Figure 2A). The second patient was treated with cetuximab-irinotecan as third line therapy for left sided metastatic CRC. A ctKRAS G12C neomutation was confirmed in further liquid biopsy and preceded radiologic disease progression (Figure 2B). Of note, an EGFR S492R mutation was found in one patient with locally advanced right-sided KRAS wildtype CRC (insufficient tumor material for NRAS and BRAF analysis). This patient received prior FOLFOX first-line therapy without anti-EGFR MoAb. In this patient, panitumumab was preferred to cetuximab as second-line therapy due to the mutation of the extracellular domain of EGFR. Panitumumab was stopped after 3 cycles due to clinical and biological (CEA) progression while CTscan stabilization was observed.

A
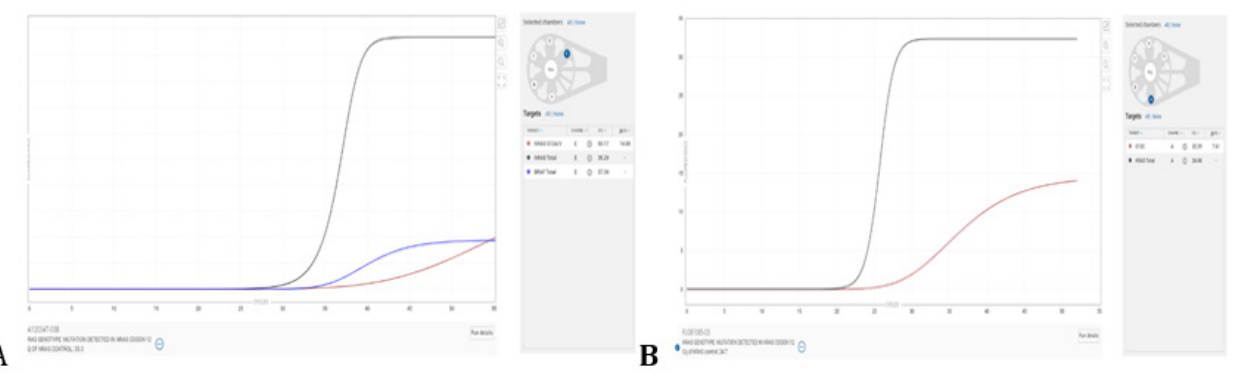

Figure 2: Visualization of neomutations PCR curves from the Idylla ${ }^{\mathrm{TM}}$ tests results.

\section{Loss of Mutation in Mutant CRC Patients}

Of the eight (16\%) patients with previously treated (i.e., at least one prior line of therapy) mutant RAS or BRAF metastatic CRC, six patients were ctRAS/RAF wild-type and two patients were ctRAS or ctBRAF mutant.

\section{Discussion}

This is the first prospective report of using the Idylla ${ }^{\mathrm{TM}}$ ctKRAS and ctNRAS/BRAF/EGFR testing for patients with advanced
CRC in daily clinical practice. Clinical research study nurse was responsible for this program. Blood sampling was located close to the translational research laboratory inorder to shorten the preanalytical time, with a median time from sample collection to the Idylla ${ }^{\mathrm{TM}}$ processing of 34 minutes, including the centrifugation process. Molecular diagnostic testing of four genes (KRAS, NRAS, BRAF and EGFR) took less than 5 hours, enabling physicians to make timely therapeutic decisions, and compare favorably with 56 days necessary to obtain a full tumor molecular evaluation.In 
the unselected population, the overall agreement between plasma and tumor was $73 \%$. Discordant resultswere observed in patients with extra-liver disease and/or in patients having received prior therapy for metastatic disease. Thus, when aiming to obtain a rapid molecular diagnosis, the Idylla ${ }^{\mathrm{TM}}$ testing should be restricted to patients with previously untreated CRC with liver metastasis, with an overall agreement rate of $100 \%$. Serial tracking of the RAS and BRAF mutation emergence in patients with wild-type CRC treated with anti-EGFR MoAb was previously described [15].

In our study, a transient ctNRAS mutation was found in one patient treated with cetuximab-based therapy, but was not associated with disease progression, whereas a confirmed ctKRAS mutation preceded failure of cetuximab-based therapy. Liquid biopsies could help to identify patients having tumors with secondary resistance to drugs and to develop different treatment strategies using agents targeting neomutations for those patients. Serial tracking of the RAS or BRAF mutation loss may also be offered to patients with mutant RAS or BRAF CRC. It has been previously suggested that patients with metastatic CRC harboring mutated primary tumors, thus not candidate to EGFR MoAb, frequently have wild-type RAS circulating tumor cells in blood [16]. Preliminary data suggest that patients with mutant KRAS CRC can frequently (50\%) switch to a prevalent wild-type KRAS disease in course of treatment with anti-angiogenic drugs [17]. This phenomenon was concomitant to disease progression. In our series, six of the eight patients with previously treated mutant RAS or BRAF metastatic CRC were ct wild-type. Those 'NeoWild-Type' patients may be eligible to anti-EGFR therapy and warrant larger prospective evaluation in this particular setting.

\section{Conclusion}

Extended RAS mutational status analysis in circulating tumor DNA from patients with advanced CRC is feasible and helpful for daily practice routine.We recommend restricting the Idylla ${ }^{\mathrm{TM}} \mathrm{ctDNA}$ testing to patients with previously untreated liver metastatic disease.

\section{Aknowledgment}

We thank Dr. Magdalena Benetkiewicz for help in editing the manuscript and Mr. Sébastien Le Maner and Ms. Nathalie Vandenbossche (Merck, France) for partnership.

\section{Key Message}

We evaluated the feasibility of plasma RAS and BRAF analysis of patients with advanced colorectal cancer in daily routine clinical practice. The Idylla ${ }^{\mathrm{TM}}$ testing provided sameday results (within 5 hours) enabling physicians to make timely therapeutic decisions. We recommend restricting ctDNA testing to patients with previously untreated liver metastatic disease.

\section{Fundings:}

The Idylla ${ }^{\mathrm{TM}}$ console was provided by Biocartis.

This program was supported by a grant from Merck France [affiliate of Merck KGaA (Darmstadt, Germany)] to Franco-British Institute, France.

\section{References}

1. Coachman C, Calamita M (2014) Virtual Esthetic Smile Design. J Cosmet Dent 29(4): 102-116.

2. Caviggioli I, Molinelli F, Ortensi L, Stefani R (2011) La prima visita in odontoiatria protesica: aspetti innovativi. Il Dentista Modernogiugno.

3. Zimmermann M, Mehl A (2015) Virtual smile design systems: a current review. Int J Computer Dent 18(4): 303-317.

4. Coachman C, Calamita M (2012) A Tool for Treatment Planning and Communication in Esthetic Dentistry QDT.

5. Goodacre CJ, Garbacea A, Naylor WP, Daher T, Marchack CB, Lowry J (2012) CAD/CAM fabricated complete dentures: concepts and clinical methods of obtaining required morphological data. J Prosthet Dent 107(1): 34-46.

6. Infante L, Yilmaz B, Mc Glumphy E, Finger I (2014) Fabricating complete dentures with CAD/CAM technology. J Prosthet Dent 111(5): 351-355.

7. Kattadiyil MT, Jekki R, Goodacre CJ, Baba NZ (2015) Comparison of treatment outcomes in digital and conventional complete removable dental prosthesis fabrications in a predoctoral setting. J Prosthet Dent 114(6): 818-825.

8. Rossi M, Molinelli F, Caviggioli I (2015) Latest trends in prosthetics-Total maxillary rehabilitation with a Toronto Bridge using digital technologies. CAD/CAM International magazine of digital dentistry 1: 28-31.

9. McLaren EA, Terry D (2001) Photography in dentistry. J Calif Dental Ass 29(10): 735-742.

10. Kim JE, Amelya A, Shin Y, Shim JS (2017) Accuracy of intraoral digital impressions using an artificial landmark. J Prosthet Dent 117(6): 755761.

11. Patzelt SB, Vonau S, Stampf S, Att W (2013) Assessing the feasibility and accuracy of digitizing edentulous jaws. The Journal of the American Dental Association 144(8): 914-920.

12. Schreinemakers J (1979) Die Logik in der Totalprothetik. Quintessenzp. 360.

13. Stefani R, Caviggioli I, Molinelli F, Ortensi L (2012) L'impiego delle tecnologie digitali nella diagnosi protesica e nella realizzazione della protesi. Il Dentista Moderno Ottobre.

14. Jokstad A (2017) Computer-assisted technologies used in oral rehabilitation and the clinical documentation of alleged advantages-A systematic review. J Oral Rehab 44(4): 261-290.

15. Lombardi RE (1977) Factors mediating against excellence in dental esthetics. J Prosthet Dent 38(3): 243-248.

16. Joda T, Brägger U, Gallucci G (2015) Systematic literature review of digital three-dimensional superimposition techniques to create virtual dental patients. Int J Oral Maxillofac Implants 30(2): 330-337.

17. Zhang C, Liu T, Liao W, Yang T, Jiang L (2017) Computer-aided design of dental inlay restoration based on dual-factor constrained deformation. Advances in Engineering Software 114: 71-84.

18. Rengier F, Mehndiratta A, von Tengg-Kobligk H, Zechmann CM, Unterhinninghofen R, et al. (2010) 3D printing based on imaging data: review of medical applications. Int J Comput Assist Radiol Surg 5(4): 335-341.

19. Burkhardt MA, Waser J, Milleret V, Gerber I, Emmert M, et al. (2016) Synergistic interactions of blood-borne immune cells, fibroblasts and extracellular matrix drive repair in an in vitro peri-implant wound healing model. Scientific reports 6: 21071.

20. Coachman C, Calamita MA, Coachman FG, Coachman RG, Sesma N (2017) Facially generated and cephalometric guided 3D digital design for complete mouth implant rehabilitation: A clinical report. J Prosthet Dent 117(5): 577-586. 
21. Koutsoukis T, Zinelis S, Eliades G, Al-Wazzan K, Rifaiy MA, et al. (2015) Selective Laser Melting Technique of Co-Cr Dental Alloys: A Review of Structure and Properties and Comparative Analysis with Other Available Techniques. Journal of Prosthodontics 24(4): 303-312.

22. Goodacre BJ, Goodacre CJ, Baba NZ, Kattadiyil MT (2018) Comparison of denture tooth movement between CAD-CAM and conventional fabrication techniques. J Prosthet Dent 119(1): 108-115.

\section{ISSN: 2574-1241}

DOI: 10.26717/BJSTR.2018.06.001409

Benoist Chibaudel. Biomed J Sci \& Tech Res

(C)

Submission Link: https://biomedres.us/submit-manuscript.php
23. Montanari M, Bonato G, Ortensi L (2016) Oral Rehabilitation with Implant-Supported Overdenture and a New Protocol for Bar Passivation. Global Journal of Oral Science 2: 10-19.

Assets of Publishing with us
- Global archiving of articles
- ImmedicAL

